\title{
Downstream Signaling of the Sos Gene is Not Required during the Pathogenesis of Cancer Cells Bearing KRAS and BRAF Mutations
}

Tamimi Yahya*, Al Busaidi Aisha, Gupta Ishita and AL Moundhri Mansour

College of Medicine \& Health sciences, Sultan Qaboos University, Muscat, Sultanate of Oman

"Corresponding author: Tamimi Yahya, Department of Biochemistry, College of Medicine \& Health Sciences, Sultan Qaboos University, Muscat, Sultanate of Oman, Tel: +96824143522; Fax: 96824413880; E-mail: yahyatam@squ.edu.com

Received date: August 28, 2015; Accepted date: October 17, 2015; Published date: October 23, 2015

Copyright: @2015 Tamimi Y, et al. This is an open-access article distributed under the terms of the Creative Commons Attribution License, which permits unrestricted use, distribution, and reproduction in any medium, provided the original author and source are credited.

\begin{abstract}
The Braf/Ras-MAPK is a relevant signal transduction pathway involved in several processes of the cell cycle through activation by interacting with several growth factors and commonly mutated in several cancers. The Sos-1 and Sos-2 (Son of sevenless homolog-1/2) guanine nucleotide exchange factors play a key role in regulating the Ras protein to enable downstream pathway activation. In this study, we investigated the effect of Sos 1 proteins on the regulation of the MAPK pathway in the presence and absence of KRAS and BRAF mutations, by monitoring the expression of the downstream proteins, MEK and ERK and their related phosphorylation forms. Our results revealed that knockdown of the Sos1 protein in cells harboring (G13D) and (G464V) mutations within KRAS and BRAF genes respectively did not affect the activation of the MAPK pathway. In contrast, cells with wild-type KRAS and BRAF exhibited decreased activation of MEK and ERK in response to Sos1 knockdown. This study suggests that MAPK pathway activation maneuvers independently of the upstream signaling from Sos1 when KRAS and BRAF mutations are present in tumor cells. Transduction of downstream signaling is likely due to mutations self-activation (gain-offunction mutations) or to the involvement of new oncoprotein involved in the onset of tumorogenesis through different pathway. Interaction of the different key players in this pathway tends to differ and can be critical for paving the pathway towards drug design and better therapy.
\end{abstract}

Keywords: Sos gene; Mutation; Pathogenesis

\section{Introduction}

The mitogen-activated protein kinase (MAPK) signal transduction pathway, also known as the Ras-Raf-MEK-ERK pathway, plays a significant role in cellular proliferation, differentiation, response to stress, apoptosis, survival and cytokine production [1,2]. Although, the importance of this pathway in transformation and tumorigenesis has been well established [3], the mechanisms underlying the regulation between Sos entity and Ras-Raf-MEK-ERK pathway is still ambiguous and controversial $[1,2,3]$.

The Ras superfamily of GTPase proteins includes 150 members, three of which (H-Ras, N-Ras and K-Ras-4A/K-Ras-4B) are most common and are collectively referred to as the p21 Ras family [4]. Ras are plasma membrane-bound proteins, active in the form of Ras-GTP and activated by guanine exchange factors (GEFs) subfamilies such as Son of Sevenless (Sos), ras-guanine nucleotide releasing factor (GRF) and ras-guanyl nucleotide releasing protein (GRP). Ligand-mediated catalyzation of the factors allows the dissociation of GDP from Ras and promotes the binding of GTP instead, thus recruiting Raf kinase to the plasma membrane for activation [4]. When assisted by GTPase activating proteins (GAPs), the Ras proteins hydrolyse GTP to GDP, resulting in its inactivation.

Mammalian cells have two functional Sos proteins, Sos1 and Sos2, characterized by a high degree of similarity but vary in size [5]. As compared to Sos1, Sos2 is larger and tends to have a stronger binding affinity to the adaptor protein, growth factor receptor-bound protein 2 (Grb2) [6]. Sos protein is activated upon binding to Ras-GDP at the Ras exchange motif (REM) domain, an allosteric site, allowing Ras to bind the catalytic CDC25 domain where GDP is dissociated and GTP is bound to the Ras protein. At this stage, Ras triggers a downstream cascade of proteins through Raf protein recruitment and activation [4].

The Raf (MAP3K) proteins (A-RAF, B-Raf and C-Raf) transduce signals to activate MEK (Map kinase extracellular signal-regulated kinase) proteins present in three different homologues; MEK1, MEK1b and MEK2, activated by phosphorylation of serine residues and contain catalytic kinase domains. These active MEKs regulate downstream effectors ERK1/2 (extracellular signal-regulated kinase) through their serine/threonine kinase domain on ERKs' tyrosine and threonine residues [2]. The active ERKs are translocated into the nucleus to phosphorylate transcription factors such as Elk1, c-Fos, p53, Est $1 / 2$ and c-Jun. Additionally, ERKs have a negative feedback activity on Sos, Raf and MEK [2].

While mutations of the Ras proteins have been found in $15-30 \%$ of all cancers, Raf mutations occur in $7 \%$ of cancers with the BRAF (V600E) variant being the most common [7-9]. Mutations in KRAS (V-Ki-ras2 Kirsten rat sarcoma viral oncogene homolog) and BRAF (vRaf murine sarcoma viral oncogenes homolog1) genes are most commonly reported in colorectal cancer (CRC), with activated KRAS and mutated BRAF accounting for $45 \%$ and $15 \%$ respectively [9-11]. Mutations in KRAS and BRAF genes are characterised by gain of function where the active form of the proteins is conserved [12]. Gainof-function mutations of these proteins, specifically KRAS, prevent the GAP proteins from hydrolysing GTP bound KRAS and therefore, remain on active mode $[13,14]$. Eversince, the epidermal growth factor receptor (EGFR) became the main focus for medical research to develop treatment of metastatic colorectal cancer (mCRC) through EGFR targeting. Responses to anti-EGFR monoclonal antibodies (cetuximab and panitummab) are predicted through the potential 
Citation: Tamimi Yahya, Al Busaidi Aisha, Gupta Ishita, AL Moundhri Mansour (2015) Downstream Signaling of the Sos Gene is Not Required during the Pathogenesis of Cancer Cells Bearing KRAS and BRAF Mutations. J Carcinog Mutagne 6: 240. doi: $10.4172 / 2157-2518.1000240$

Page 2 of 7

biomarkers; KRAS and BRAF [15]. KRAS mutations are considered as "negative predictive factors" as they are resistant to the EGFR-targeted monoclonal antibodies treatments. Moreover, a slight role in resistance to anti-EGFR was observed with BRAF mutated mCRCs [16]. Although, Sos1 gain-of-function mutations have been observed in disorders such as the Noonan syndrome and hereditary gingival fibromatosis $[17,18]$ as well as a few tumors including pancreatic, lung and prostate cancers $[19,20]$, their underlying mechanisms still remain nascent.

Considering the critical role played by Sos in activating the Ras protein, it is important to understand its differential role in tumours with mutations in the MAPK pathway. Moreover, compounds targeting the interaction between Ras and Sos are being investigated to inhibit the MAPK pathway in tumours [21] and the efficacy of such compounds relies on the dependence of the mutant Ras pathway on Sos function. In the present study, we investigated the role of Sos protein in activating the MAPK pathway in cancers with oncogenic Ras and Raf proteins by Sos1 knockdown using both, colorectal and breast cancer cell lines with different mutation status. Our findings indicate that oncogenic Ras activates the downstream MAPK pathway independently of Sos, thus suggesting other compounds within or from other pathways to be involved in the oncogenic Ras activation in the presence of Sos.

\section{Materials and Methods}

\section{Cell lines}

Two colorectal cancer cell lines (HT-29 and Caco-2) and one breast cancer cell line (MDA-MB-231) were used for this study. HT-29 and Caco-2 (epithelial-adenocarcinoma), (purchased from ATCC, Manassas, VA) and MDA-MB-231 (breast epithelial adenocarcinoma) cells (obtained from Dr. R Godbout, Cancer Research Institute, University of Alberta, Canada). Cells were maintained and propagated in Dulbecco's Modified Eagle Medium (DMEM) supplemented with $10 \%$ foetal bovine serum (FBS) and with $1 \%$ penicillin-streptomycin and in a humidified incubator at $37^{\circ} \mathrm{C}$ and $5 \%$ of $\mathrm{CO}_{2}$ atmosphere.

\section{siRNA Treatment}

Total RNA was isolated from the cells using RNeasy Mini kit (Qiagen) according to the manufacturer's instructions. Predesigned human Sos1 siRNA oligonucleotides were obtained from Cell Signaling Technology and cells were transfected according to the manufacturer's instructions. Cells were cultured in 6-well plates in antibiotic-free DMEM (Gibco) prior to transient transfection with small interfering RNA (siRNA) (Cell Signaling) to shutdown Sos1 expression. The siRNA for each mRNA is a mixture of three different siRNA acting on different positions along the mRNA at a final concentration of $27 \mu \mathrm{M}$ and incubated at room temperature for 5 minutes. Lipofectamine RNAi MAX, a cationic lipid transfecting reagent (Invitrogen) was used as a vector to carry siRNA inside the cells. The cells were subsequently incubated at $37^{\circ} \mathrm{C}$ with an atmosphere of $5 \% \mathrm{CO}_{2}$ for $15,18,24$ and 48 hours.

\section{Preparation of protein samples}

Cells were lysed in lysis buffer (composed of 20 mMTris Buffer pH7.5, $50 \mathrm{mM} \mathrm{NaCl}, 1 \mathrm{mM}$ EGTA, 1 \%Triton X100, $2.5 \mathrm{mM}$ Sodium pyrophosphate, $1 \mathrm{mM}$ Sodium orthovanadate) containing $1 \mathrm{X}$ protease inhibitor (100X Halt Protease Inhibitor Cocktail, Thermo Scientific).
Orthovanadate was supplemented to the lysis buffer to inhibit phosphatases. The final protein concentration in the supernatant was determined using the bradford protein assay reagent (Pierce, Rockford, IL) and absorbance was read at $595 \mathrm{~nm}$.

\section{Western blot analysis}

$20 \mu \mathrm{g}$ of protein lysates were boiled for $5 \mathrm{~min}$ in an equal volume of reducing buffer, resolved on $12 \%$ polyacrylamide gels, and electroblotted onto nitrocellulose membranes. Membranes were probed human polyclonal anti-Sos1, (1:2000 dilution, Santa Cruz, CA), monoclonal anti- $\beta$-actin, monoclonal anti-MEK, monoclonal antipMEk, monoclonal anti-ERK and monoclonal anti-pERK (1:2000 dilution, Cell Signaling, CA), followed by a anti-rabbit polyclonal (1:4000 dilution, Santa Cruz, CA) secondary antibody. Immunoreactivity was detected using chemiluminescence as recommended by the manufacturer (Amersham Health Sciences).

\section{PCR amplification and sequencing}

Cells were grown to 70-80\% confluency and DNA was isolated from the cultured cells using the Blood and Cell Culture DNA Mini Kit (QIAGEN) according to the manufacturer's instructions. The extracted DNA was quantified by using the Nano drop spectrophotometer.

Specific primers targeting hot spot areas of KRAS (Exons 1-4), BRAF (Exons 10-13 and 15-16) and Ras motif binding domains for Sos1 (Exons 11-15) gene, was designed using Primer3 software (PCR primer design tool) (v.0.4.0) using the required DNA sequence as a template (Tables 1 and 2). GAPDH (Glyceraldehyhe 3-phosphate dehydrogenase) was used as a control. Amplification included a 5-min denaturation set up at $95^{\circ} \mathrm{C}$, followed by 35 cycles each consisting of 50s denaturation at $94^{\circ} \mathrm{C}, 50 \mathrm{~s}$ of annealing at temperatures ranging from 58 to $65^{\circ} \mathrm{C}$ depending on each exon's (melting temperature), and $50 \mathrm{~s}$ extension at $72^{\circ} \mathrm{C}$. After the last cycle, the samples were incubated for $7 \mathrm{~min}$ at $72^{\circ} \mathrm{C}$ for final extension. The PCR product from each exon was visualized using $1.5 \%$ agarose gel electrophoresis.

\begin{tabular}{|c|c|c|c|}
\hline Primer & Primer & $\begin{array}{l}\text { Product } \\
\text { Size }\end{array}$ & $\begin{array}{l}\text { Working } \\
\operatorname{Tm}\left({ }^{\circ} \mathrm{C}\right)\end{array}$ \\
\hline KRAS-Ex-1F & 5'CT CGGAGCTCGATTTTCCTA-3' & \multirow[b]{2}{*}{$296 \mathrm{bp}$} & \multirow[b]{2}{*}{56.0} \\
\hline $\begin{array}{l}\text { KRAS- } \\
\text { Ex-1R }\end{array}$ & 5'-GGGGACCCCTAATTCATTCA-3 & & \\
\hline KRAS-Ex-2F & $\begin{array}{l}\text { 5'- } \\
\text { TTAACCTTATGTGTGACATGTTCTAA-3' }\end{array}$ & \multirow{2}{*}{$224 \mathrm{bp}$} & \multirow{2}{*}{57.0} \\
\hline $\begin{array}{l}\text { KRAS- } \\
\text { Ex-2R }\end{array}$ & 5'-AGAATGGTCCTGCACCAGTAA-3' & & \\
\hline KRAS-Ex-3F & 5'-TTCAAGTCCTTTGCCCATTT-3' & \multirow{2}{*}{$376 \mathrm{bp}$} & \multirow[b]{2}{*}{56.0} \\
\hline $\begin{array}{l}\text { KRAS- } \\
\text { Ex-3R }\end{array}$ & 5'-TGCATGGCATTAGCAAAGAC-3' & & \\
\hline KRAS-Ex-4F & $\begin{array}{l}\text { 5'- } \\
\text { GATATTTGTGTTACTAATGACTGTGCT- } \\
\text { 3' }\end{array}$ & \multirow[t]{2}{*}{$390 \mathrm{bp}$} & \multirow[t]{2}{*}{55.0} \\
\hline $\begin{array}{l}\text { KRAS- } \\
\text { Ex-4R }\end{array}$ & 5'-AAGAAGCAATGCССТСТCAA-3' & & \\
\hline $\begin{array}{l}\text { BRAF- } \\
\text { Ex-10F }\end{array}$ & 5'-AACATACTTGCTCCTCCTTAATGT-3' & $261 \mathrm{bp}$ & 58.0 \\
\hline
\end{tabular}


Citation: Tamimi Yahya, Al Busaidi Aisha, Gupta Ishita, AL Moundhri Mansour (2015) Downstream Signaling of the Sos Gene is Not Required during the Pathogenesis of Cancer Cells Bearing KRAS and BRAF Mutations. J Carcinog Mutagne 6: 240. doi: $10.4172 / 2157-2518.1000240$

Page 3 of 7

\begin{tabular}{|c|c|c|c|}
\hline $\begin{array}{l}\text { BRAF- } \\
\text { Ex-10R }\end{array}$ & $\begin{array}{l}\text { 5'- } \\
\text { AAGGATAATATTACATTTGGCTGTGA-3' }\end{array}$ & & \\
\hline $\begin{array}{l}\text { BRAF- } \\
\text { Ex-11F }\end{array}$ & 5'- TTTCTTTTTCTGTTTGGCTTGA-3' & \multirow{2}{*}{$199 \mathrm{bp}$} & \multirow{2}{*}{54.0} \\
\hline $\begin{array}{l}\text { BRAF- } \\
\text { Ex-11R }\end{array}$ & 5'- ACTTGTCACAATGTCACCACA-3' & & \\
\hline $\begin{array}{l}\text { BRAF- } \\
\text { Ex-12F }\end{array}$ & 5'-TTAGTGAAAACACCCAAGAATGT- 3' & \multirow{2}{*}{$232 \mathrm{bp}$} & \multirow{2}{*}{57.0} \\
\hline $\begin{array}{l}\text { BRAF- } \\
\text { Ex-12R }\end{array}$ & 5'-CACTGGGAACCAGGAGCTAA- 3' & & \\
\hline $\begin{array}{l}\text { BRAF- } \\
\text { Ex-13F }\end{array}$ & $\begin{array}{l}\text { 5'- } \\
\text { GAATTGCTAAAGTTTGTCGACATTTT-3 }\end{array}$ & \multirow{2}{*}{$315 \mathrm{bp}$} & \multirow{2}{*}{54.0} \\
\hline $\begin{array}{l}\text { BRAF- } \\
\text { Ex-13R }\end{array}$ & 5'-TCCAAAAGAATAGCAGCCAAA-3' & & \\
\hline $\begin{array}{l}\text { BRAF- } \\
\text { Ex-15F }\end{array}$ & 5'-TGCTTGCTCTGATAGGAAAATG- 3' & \multirow{2}{*}{$227 \mathrm{bp}$} & \multirow{2}{*}{55.0} \\
\hline $\begin{array}{l}\text { BRAF- } \\
\text { Ex-15R }\end{array}$ & 5'-AGCATCTCAGGGCCAAAAAT- 3' & & \\
\hline $\begin{array}{l}\text { BRAF- } \\
\text { Ex-16F }\end{array}$ & $\begin{array}{l}\text { 5'- } \\
\text { AGGTTGTTTTTCAGTCTTTATTCAA-3, }\end{array}$ & \multirow{2}{*}{$258 \mathrm{bp}$} & \multirow{2}{*}{55.0} \\
\hline $\begin{array}{l}\text { BRAF- } \\
\text { Ex-16R }\end{array}$ & 5'-TTTTCTACAACTGGAGCCTTG-3' & & \\
\hline $\begin{array}{l}\text { GAPDH- } \\
\text { Ex-8-F }\end{array}$ & 5'-GGCTCTCCAGAACATCATCC-3' & \multirow{2}{*}{$240 \mathrm{bp}$} & \multirow{2}{*}{56.0} \\
\hline $\begin{array}{l}\text { GAPDH- } \\
\text { Ex-8-R }\end{array}$ & 5'-ACCTGGTGCTCAGTGTAGCC-5' & & \\
\hline
\end{tabular}

Table 1: Primers to amplify exons of the KRAS and BRAF genes.

\begin{tabular}{|c|c|c|c|}
\hline Primer & Primer & $\begin{array}{l}\text { Product } \\
\text { Size }\end{array}$ & $\begin{array}{l}\text { Working } \\
\operatorname{Tm}\left({ }^{\circ} \mathrm{C}\right)\end{array}$ \\
\hline SOS1-Ex-11F & $\begin{array}{l}\text { 5'-; CATGAGCTCTAGGTTTTCTGTCA } \\
-33^{\prime}\end{array}$ & \multirow[t]{2}{*}{$840 \mathrm{bp}$} & \multirow[t]{2}{*}{59.0} \\
\hline SOS1-Ex-11R & 5'- TTAGGCACAATAAACCCATGC -3' & & \\
\hline SOS1-Ex-12F & 5'- GCAGTGCATTACCAAGTCCA - 3' & \multirow{2}{*}{$333 \mathrm{bp}$} & \multirow{2}{*}{57.0} \\
\hline SOS1-Ex-12R & $\begin{array}{l}\text { 5'- ATTTATTGAAAAAGTGCTTGTGAA } \\
-33^{\prime}\end{array}$ & & \\
\hline SOS1-Ex-13F & 5'- TTTGCTGACTGGTGAAAACG- 3' & \multirow{2}{*}{$313 \mathrm{bp}$} & \multirow{2}{*}{57.0} \\
\hline SOS1-Ex-13R & 5'- TTGTCACCССTCTCCTTGTT- 3' & & \\
\hline SOS1-Ex-14F & $\begin{array}{l}\text { 5'- TTTGGAAGTGTTAAGCACACTGA- } \\
3^{\prime}\end{array}$ & \multirow{2}{*}{$351 \mathrm{bp}$} & \multirow{2}{*}{59.0} \\
\hline SOS1-Ex-14R & 5'- TTACTGAGCCCCAATGACATC- 3' & & \\
\hline SOS1-Ex-15F & 5'- CATTCAGGTGTCATCCGTGT- 3' & \multirow{2}{*}{$462 \mathrm{bp}$} & \multirow{2}{*}{59.0} \\
\hline SOS1-Ex-15R & 5'-GCCTGCCTGGCCTTATTACT- 3' & & \\
\hline
\end{tabular}

Table 2: Primers to amplify exons of the Sos1 gene.

To screen for mutations in KRAS, BRAF and Ras motif binding domains for Sos1 gene, PCR products were sequenced using the ABI BigDye Terminator v3.1 Cycle Sequencing Kit. The conditions of the

sequencing reaction included 25 cycles at $96^{\circ} \mathrm{C}(10 \mathrm{~s}), 60^{\circ} \mathrm{C}(5 \mathrm{~s}), 60(4$ $\mathrm{min}), 4^{\circ} \mathrm{C}$ (holding temperature). The sequencing data was analysed by using BioEdit sequence Alignment Editor Version 7.0.4.1 software and Sequencher 4.10.1 Demo software.

\section{Results}

\section{Confirmation of the presence/absence of BRAF and KRAS mutations by sequence analysis}

In order to confirm the previously reported mutations in the BRAF, KRAS and screening for eventual mutations in Sos1 gene, PCR amplified products of the targeted exons were sequenced using Sanger's (R) method. Sequences were aligned with the correspondent wild type reference sequences found on public data bases such as Ensemble Genome Browser/Havana merge, using the Sequencher software.

In MDA-MB-231 cells, KRAS exon 2 sequence showed a substitution of base $G$ to $A$ at codon 13 , changing the amino acid Glycine to Aspartic Acid; G13D (GGC>GAC). This mutation confirms the previously reported mutation of KRAS gene in breast cancer cell line MDA-MB-231. However, in the colon cancer cell lines, HT-29 and Caco-2 no mutation was found in exons 2, 3 and 4 of the KRAS gene.

In Caco- 2 cell line, BRAF sequence analysis, revealed no changes in any of the exons examined. Whereas, analysis of exon 11 in BRAF showed a substitution of $\mathrm{G}$ to $\mathrm{T}$ base (GGA>GTA) at codon 464 in MDA-MB-231 cells, changing the amino acid Glycine to Valine. Moreover, BRAF sequence analysis in HT-29 cells confirmed the previously reported common BRAF mutation in exon 15, codon 600 changing the amino acid Valine to Glutamic Acid (V600E).

\section{Western Blot results for Sos protein expression}

Non-transfected HT-29, Caco2 and MDA-MB-231 cells were assessed for their Sos expression levels. Both, Caco-2 and MDAMB-231 cells displayed a clear band of the expected size (155kDa) related to Sos1 (Figure 1). However, in comparison to Caco-2 and MDA-MB-231 cells, HT-29 cell line displayed a very faint band. $\beta$ actin $(45 \mathrm{kDa})$ was used as a loading control (Figure 1$)$.

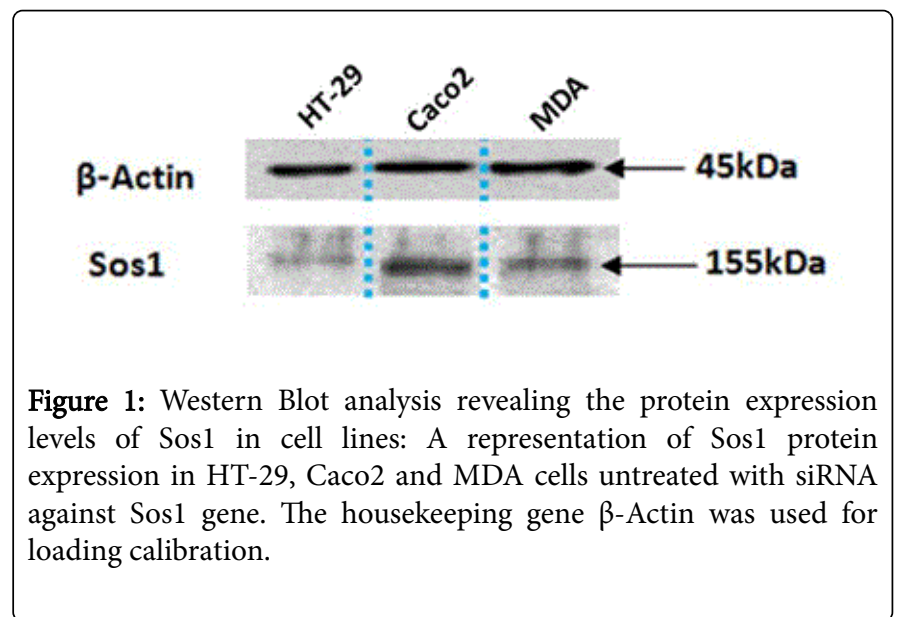

\section{siRNA treatment to knock down Sos1 gene}

In order to determine the most efficient time interval for transfection, cell lines were transfected for 15,18 and 48 hours prior to 
Citation: Tamimi Yahya, Al Busaidi Aisha, Gupta Ishita, AL Moundhri Mansour (2015) Downstream Signaling of the Sos Gene is Not Required during the Pathogenesis of Cancer Cells Bearing KRAS and BRAF Mutations. J Carcinog Mutagne 6: 240. doi: $10.4172 / 2157-2518.1000240$

Page 4 of 7

cell harvest and protein analysis. $\beta$-Actin was uniformly expressed in all the various conditions applied.

In the Caco-2 cells, after 15 hours of siRNA transfection (Group A), Sos1 protein expression was inhibited by only $10 \%$ (Figure 2 ).

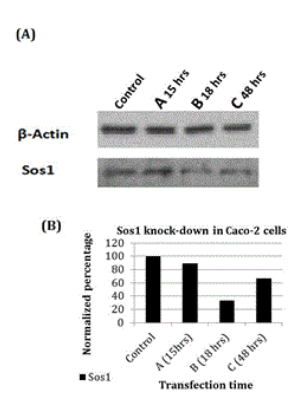

Figure 2: siRNA efficiency to knock down Sos1 gene in Caco-2 cells. (A) Western Blot showing the effect of siRNA treatment at different time intervals on Sos1 protein expression in the Caco-2 colorectal cancer cell line. CTRL represents the untreated control sample. $\beta$ Actin expression was utilized as housekeeping gene for calibration. (B) Quantification of the Sos1 knockdown efficiency at the protein level using an image analysis software "the Image J software". Sos1 expression is normalized to the corresponding level of $\beta$-Actin and represented as a percentage of the Sos1 expression in the control sample.

However, after 18 hours of siRNA transfection, Sos1 expression was significantly inhibited (Group B). Prolonged transfection time (Group C-48 hours) resulted in the Sos1 protein levels trending towards the control levels.

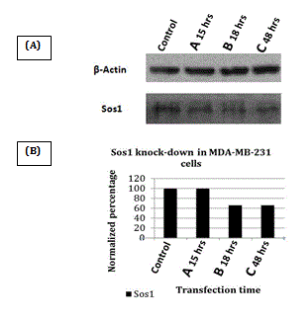

Figure 3: siRNA efficiency to knock down Sos1 gene in MDAMB-231 cells. (A) Western Blot showing the effect of siRNA treatment at different time intervals on Sos1 protein expression in the MDAMB-231 breast cancer cell line. CTRL represents the untreated control sample. $\beta$-Actin expression was utilized as a housekeeping gene for loading calibration. (B) Quantification of the efficiency of Sos1 knockdown at the protein level using western blot and an image analysis software "the Image J software". Sos1 expression is normalized with the corresponding level of $\beta$-actin and represented as a percentage of the Sos1 expression in the control sample.

Interestingly, in the MDA-MB-231 cell line as well, Sos1 knockdown was most significant after an 18-hour transfection period and reduced protein levels were maintained during the 48 -hour transfection period (Figure 3).
Contrary to the other cell lines, HT-29 cells indicated no changes in Sos1 protein expression for all the transfection periods applied as compared to the non-transfected control, which showed lower levels of Sos1 expression (Figure 4).

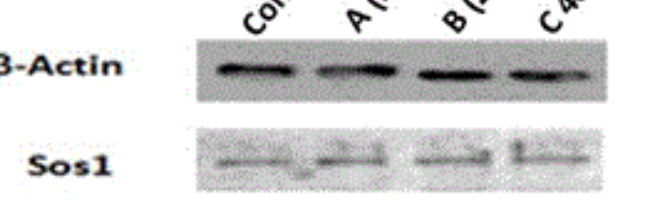

Figure 4: siRNA efficiency to knock down Sos1 gene in HT-29 cells. Western Blot showing the effect of siRNA treatment at different time intervals on Sos1 protein expression in the HT-29 colorectal cancer cell line.

\section{The effect of Sos1 knockdown on the downstream effectors in the MAPK pathway}

To assess the effect of Sos1 knockdown on downstream signalling, total protein extracted from cells collected at different transfection time periods were analysed for the expression levels of MEK and ERK proteins. In the Caco-2 cell line, Sos1 knockdown did not affect the total MEK and ERK protein level (Figure 5).

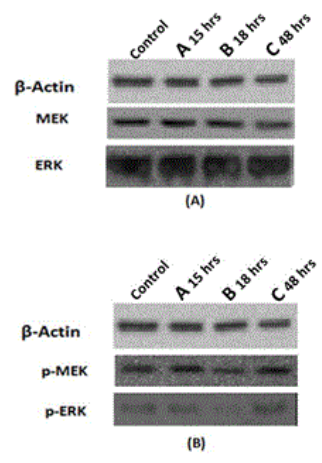

Figure 5: MEK and ERK protein expressions following siRNA knock down of Sos1 gene in Caco-2 cells. A) Western Blot showing the effect of siRNA treatment at different time intervals on MEK and ERK protein expressions. (B) Western Blot showing the effect of Sos1 siRNA treatment at different time intervals on active phosphoMEK and phospho-ERK protein expressions. CTRL represents the untreated control sample. $\beta$-Actin expression was utilized as a housekeeping gene for calibration.

However, the level of the active phosphorylated form of both MEK and ERK were most significantly reduced after an 18-hour transfection period when Sos1 protein level was knocked down by 50\% (Figure 5). Contrastingly, in the MDA-MB-231 cells, no changes in total and phosphorylated MEK and ERK protein were observed despite the $20 \%$ knockdown in Sos1 protein level after 24 or 48 hours of transfection (Figure 6). 


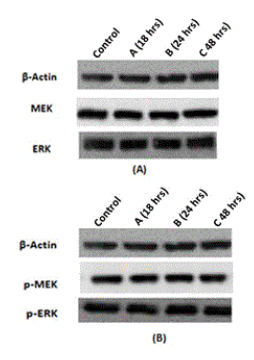

Figure 6: MEK and ERK protein expressions following siRNA knock down of Sos1 gene in MDA-MB-231 cells. (A) Western Blot showing the effect of siRNA treatment at different time intervals on MEK and ERK protein expressions. (B) Western Blot showing the effect of Sos1 siRNA treatment at different time intervals on active phospho-MEK and phospho-ERK protein expressions. CTRL represents the untreated control sample. $\beta$-Actin expression was utilized as a housekeeping gene for calibration.

As expected, HT-29 cells that did not display any knockdown of Sos1 protein, total protein levels and phosphorylated MEK and ERK were similar to the untreated control levels (Figure 7).

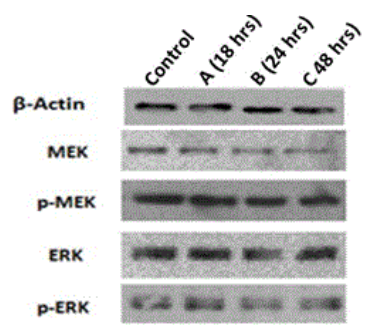

Figure 7: MEK and ERK protein expressions following siRNA knock down of Sos1 gene in HT-29 cell line. Western Blot showing the effect of siRNA treatment at different time intervals in the HT-29 cells on the protein expression of MEK, phosphor MEK, ERK, and phosphor ERK. CTRL represents the untreated control sample. $\beta$-Actin expression was utilized as a housekeeping gene for calibration control.

\section{Discussion}

There are two major cellular pathways in which Ras proteins are involved, MAPK and PI3K pathways. In this study, we have focused on the MAPK pathway, a fundamental cascade that controls cell cycle progression, apoptosis and differentiation [22]. KRAS and BRAF, key members of the MAPK pathway, are most commonly mutated in cancers, particularly in colorectal cancer [23]. KRAS mutations for instance are found in $40 \%$ and BRAF mutations in 5 to $10 \%$ of all colorectal cancers [11]. KRAS and BRAF mutations are known to be involved in the malignant transition of colon cancer [24]. To activate this pathway, Sos needs to bind Ras, thus displacing GDP from Ras and permitting GTP to bind Ras protein [4]. Sos proteins have two domains; the Cdc25 domain that allows stabilization of the protein and the REM domain that promotes binding and subsequent activation of Ras proteins. REM domain exhibits also an allosteric binding with Ras to induce its own activation [25].
Sos1 has been described as a gene with polyvalent function involved in key functions of the cell cycle and the related diseases such as cancer. Sos 1 is responsible for driving constitutive activation of NF kappa B and binds Abil in pancreatic cancer [26]. It was also reported that Sos 1 is targeted by miR-146a and miR-370 to induce cell apoptosis and it gain of function mutations was associated with a distinctive form of Noonan syndrome [27,28].

The effect of Sos proteins, have not been well understood in cancers and very few related studies were reported in colorectal cancer [28]. Therefore, in this study we aimed to shed some light on the effect of Sos proteins on MAPK pathway in colorectal cancers, and used HT-29 cells, known to carry mutations in BRAF gene, and Caco- 2 cells reported to display no mutations in KRAS or BRAF. Additionally, to compensate for the mutual exclusivity of KRAS and BRAF mutations in colon cancer cell lines, the breast cancer cell line MDA-MB-231 known to carry both KRAS and BRAF mutations [29] was also analyzed. In the present study, we have shown that although, Sos is a transcriptional target of the wild-type Ras and Braf oncogene in colon cancer, this regulation has no effect in the presence of activating mutation in the downstream gene. Activation of KRAS has been known to be responsible for signal transduction via multiple pathways, leading to several cancer characteristics including reduced cell death and increased cell growth and progression. In this study we have shown that Sos is dispensable in cancer cells carrying KRAS and /or BRAF mutations and transduction of downstream signaling is carried out by self-activation mutations (gain-of-function mutations) or by recruiting other oncoproteins involved in the onset of tumorogenesis through different pathways.

In this study, we found that MDA-MB-231 cells successfully knocked-down for Sos1 expression and harboring both KRAS and BRAF mutations did not change the expression or activation of the downstream effectors in the MAPK signaling pathway. In contrast, Caco-2 cells with no mutations in KRAS and BRAF had reduced active phosphorylated forms of both MEK and ERK expression following a successful Sos1 knockdown in these cells. Interestingly, we showed that depletion of Sos1 in cancer cells harboring KRAS (G13D) and BRAF (G464D) mutation is not necessary to sustain the MAPK pathway activation. In MDA-MB-231 cells, sequence analysis confirmed the mutation in exon 2 of KRAS gene at codon 13, where a transition from guanine to adenine results in a Glycine to Aspartic acid (G13D) residue change. This gain-of-function mutation is similar to a guanine to thymine transversion mutation in codon 12 of the KRAS exon 2 causing a glycine to valine amino acid change [30]. These mutations cause impaired GTPase activity of the GAP proteins, where K-Ras binds to GAP proteins without activating it and thus, remain constitutively active; explaining the reported resistance of KRAS mutated CRCs to EGFR therapies such as cetuximab and panitumumab [31]. KRAS mutations at codon 12 were reported to inhibit apoptosis, correlate with aggressive phenotype and recurrence with poor prognosis and shorter overall survival [32]. Likewise, mutations at codon 13 were found to have the same effect as mutation in codon 12 but to a lesser extent [33]. B-Raf protein has been reported to have 30 single site missense gain-of-function mutations generally located in the kinase domain with elevated kinase activity and can be subdivided into three groups; high, intermediate and impaired activity [34]. The BRAF mutation in codon 464 found in MDA-MB-231 cell line is a missense mutation, resulting in a change from Glycine to Valine (G464V) and is considered as an intermediate activity mutation with up to 100 -fold higher kinase activity compared to wild-type B-Raf [35]. However, the activity and transformation potency of G464V is 
less compared to the widely prevalent, high activity V600E BRAF mutation in exon 15 present in HT-29 cells causing a Valine to Glutamic acid change. This explains the coexistence of the G464V BRAF mutation along with the G13D KRAS mutation resulting in a two-hit activation of the Ras pathway. The V600E BRAF mutation has a basal kinase activity of 700 folds higher than the activity of RAS G12V (a high active Ras mutant) [34] and hence, is mutually exclusive to Ras mutations in breast and colon cancers. The observation in this study that the downstream proteins MEK and ERK and their respective active forms were unaffected despite the knockdown of the Sos1 MDA-MB-231 cells indicates the constitutive activation of the Ras pathway due to the presence of the B-Raf and Ras mutations in this cell line, irrespective of the activity of Sos1. Mutant K-Ras (G12V) has been shown to promote the activation of wild type H-Ras and NRas by Sos-mediated allosteric stimulation [36]. Hence, decreased activation of the Ras pathway could be expected upon Sos knockdown. However, no differences in downstream pathway activation were observed between the control MDA-MB-231 cells and Sos knockdown cells, suggesting either the inability of the G13D KRAS mutation to activate wild type Ras similar to the G12D mutation or, the presence of Sos protein, albeit at lower levels, due to its incomplete knockdown in the MDAMB-231 cells can maintain pathway activation via wild-type Ras. Hence, studies investigating cellular responses and molecular changes in response to the complete knockout of Sos will provide a clearer picture of the role of Sos in Ras mutant tumor cells. Additionally, Sos independent non-canonical pathways may be directly involved in activating MEK and ERK, such as the phosphatidylinositol-3-kinases (PI-3K)-Rac1 cascade [37-41], cyclic AMP pathway via the action of Rap1 [38], protein kinase C delta activation [42] and other modulators such as kinase suppressor of Ras (KSR) and MEK-1 Partner 1 (MP1) [43]. The analysis of these pathways to elucidate their contribution in MAPK pathway activation in the presence of Ras mutations is the aim for future experiments. Interestingly, the knockdown of Sos1 protein in Caco-2 cells which lacks mutations in the KRAS and BRAF genes, affected the downstream signal transduction of this pathway, as indicated by the reduced activation of MEK and ERK proteins.

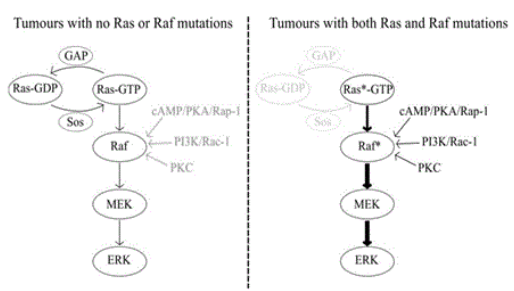

Figure 8: A schematic representation of a suggested pathway followed during activation of the MAPK pathway in cancers harboring or not Ras and Raf mutations.

However, the Sos 1 knockdown did not seem to have a direct effect on the expression of inactive forms of MEK and ERK, as their levels of expression remained equal to the levels of MEK and ERK in the Caco-2 control (untreated) cells. These data are in agreement with observations from Sos1 inhibition in other cancer cells, mice and $C$. elegans This indicates that Sos1 is the main regulator of the Ras protein, involved in the downstream MAPK pathway in cells not harboring mutations in either the Ras or Raf proteins [6,44]. Hence, the regulation of the MAPK through alternative pathways such as the cAMP, PI3K and PKA in these cancers is limited (Figure 8).

We were unable to knockdown the Sos1 expression in HT-29 cells, despite conducting several assays under different conditions. Sos1 is weakly expressed in HT-29 cell line as compared to its expression in Caco-2 and MDA-MB-231 cells. This results in an increase of ERK activation in highly active B-Raf mutants in cells such as HT-29. Similar to ERK activation, the V600E BRAF mutation carried by HT-29 cells triggers a negative feedback leading to Sos phosphorylation, dissociation from the Grb complex and inhibition [34,45-47].

In conclusion, we have provided evidence that oncogenic Ras activates the downstream MAPK pathway independently of Sos regulation. However, cancers with wild type Ras and B-raf are dependent on Sos expression to trigger the MAPK pathway, reported to be, along with PI3K, critical in the onset and progression of colorectal cancer [16].

This could be an explanation behind several anti-EGFR therapy failures in response to KRAS mutations indicating that other genes might be responsible. Thus, it is likely that compounds interfering with the interaction between Ras and Sos [21] will probably be more effective in tumors not harboring Ras or B-raf mutants. This suggests that KRAS and BRAF mutations can be used as negative biomarkers to categorize patients. More research using human samples from patients affected with colorectal cancer would certainly help to pave the way for better therapeutic strategies.

\section{Acknowledgement}

This work was supported by Sultan Qaboos University internal grant under a project No. IG/MED/BIOC/13/02

\section{Conflict of Interest}

The authors declare that there is no conflict of interest.

\section{References}

1. Wimmer R, Baccarini M (2010) Partner exchange: protein-protein interactions in the Raf pathway. Trends Biochem Sci 35: 660-668.

2. Shaul YD, Seger R (2007) The MEK/ERK cascade: from signaling specificity to diverse functions. Biochim Biophys Acta 1773: 1213-1226.

3. Dhillon AS, Hagan S, Rath O, Kolch W (2007) MAP kinase signalling pathways in cancer. Oncogene 26: 3279-3290.

4. Buday L, Downward J (2008) Many faces of Ras activation. Biochim Biophys Acta 1786: 178-187.

5. Rojas JM, Oliva JL, Santos E (2011) Mammalian son of sevenless Guanine nucleotide exchange factors: old concepts and new perspectives. Genes Cancer 2: 298-305.

6. Esteban LM, Fernández-Medarde A, López E, Yienger K, Guerrero C, et al. (2000) Ras-guanine nucleotide exchange factor sos 2 is dispensable for mouse growth and development. Mol Cell Biol 20: 6410-6413.

7. Dhomen N, Marais R (2007) New insight into BRAF mutations in cancer. Curr Opin Genet Dev 17: 31-39.

8. Emuss V, Garnett M, Mason C, Marais R (2005) Mutations of C-RAF are rare in human cancer because C-RAF has a low basal kinase activity compared with B-RAF. Cancer Res 65: 9719-9726.

9. Seth R, Crook S, Ibrahem S, Fadhil W, Jackson D, et al. (2009) Concomitant mutations and splice variants in KRAS and BRAF demonstrate complex perturbation of the Ras/Raf signalling pathway in advanced colorectal cancer. Gut 58: 1234-1241. 
Citation: Tamimi Yahya, AI Busaidi Aisha, Gupta Ishita, AL Moundhri Mansour (2015) Downstream Signaling of the Sos Gene is Not Required during the Pathogenesis of Cancer Cells Bearing KRAS and BRAF Mutations. J Carcinog Mutagne 6: 240. doi: $10.4172 / 2157-2518.1000240$

Page 7 of 7

10. Harada K, Hiraoka S, Kato J, Horii J, Fujita H, et al. (2007) Genetic and epigenetic alterations of Ras signalling pathway in colorectal neoplasia: analysis based on tumour clinicopathological features. Br J Cancer 97: 1425-1431.

11. Zenonos K, Kyprianou K (2013) RAS signaling pathways, mutations and their role in colorectal cancer. World J Gastrointest Oncol 5: 97-101.

12. Azzoni C, Bottarelli L, Campanini N, Di Cola G, Bader G, et al. (2007) Distinct molecular patterns based on proximal and distal sporadic colorectal cancer: arguments for different mechanisms in the tumorigenesis. Int J Colorectal Dis 22: 115-126.

13. Normanno N, Tejpar S, Morgillo F, De Luca A, Van Cutsem E, et al. (2009) Implications for KRAS status and EGFR-targeted therapies in metastatic CRC. Nat Rev Clin Oncol 6: 519-527.

14. Stephen AG, Esposito D, Bagni RK, McCormick F (2014) Dragging ras back in the ring. Cancer Cell 25: 272-281.

15. Vokes EE, Chu E (2006) Anti-EGFR therapies: clinical experience in colorectal, lung, and head and neck cancers. Oncology (Williston Park) 20: $15-25$.

16. Rizzo S, Bronte G, Fanale D, Corsini L, Silvestris N, et al. (2010) Prognostic vs predictive molecular biomarkers in colorectal cancer: is KRAS and BRAF wild type status required for anti-EGFR therapy?. Cancer Treat Rev 36: S56.

17. Hart TC, Zhang Y, Gorry MC, Hart PS, Cooper M, et al. (2002) A mutation in the SOS1 gene causes hereditary gingival fibromatosis type 1 . Am J Hum Genet 70: 943-954.

18. Tartaglia M, Pennacchio LA, Zhao C, Yadav KK, Fodale V, et al. (2007) Gain-of-function SOS1 mutations cause a distinctive form of Noonan syndrome. Nat Genet 39: 75-79.

19. Swanson KD, Winter JM, Reis M, Bentires-Alj M, Greulich H, et al. (2008) SOS1 mutations are rare in human malignancies: implications for Noonan Syndrome patients. Genes Chromosomes Cancer 47: 253-259.

20. Timofeeva OA, Zhang X, Ressom HW, Varghese RS, Kallakury BV, et al. (2009) Enhanced expression of SOS1 is detected in prostate cancer epithelial cells from African-American men. Int J Oncol 35: 751-760.

21. Maurer T, Garrenton LS, Oh A, Pitts K, Anderson DJ, et al. (2012) Smallmolecule ligands bind to a distinct pocket in Ras and inhibit SOSmediated nucleotide exchange activity. Proc Natl Acad Sci U S A 109: 5299-5304.

22. Steelman LS, Pohnert SC, Shelton JG, Franklin RA, Bertrand FE, et al (2004) JAK/STAT, Raf/MEK/ERK, PI3K/Akt and BCR-ABL in cell cycle progression and leukemogenesis. Leukemia 18: 189-218.

23. Chang EH, Gonda MA, Ellis RW, Scolnick EM, Lowy DR, et al. (1982) Human Genome Contains Four Genes Homologous to Transforming Genes of Harvey and Kirsten Murine Sarcoma Viruses. Proc Natl Acad Sci 79: 4848-4852.

24. Fearon ER (2011) Molecular Genetics of Colorectal Cancer. Annu Rev Pathol 6: 479-507.

25. Freedman TS, Sondermann H, Kuchment O, Friedland GD, Kortemme T, et al. (2009) Differences in flexibility underlie functional differences in the Ras activators son of sevenless and Ras guanine nucleotide releasing factor 1. Structure 17: 41-53.

26. Kiely P, Tod J, Jenei V, Johnson C, Thomas GJ (2013) The role of the Eps8 binding partners Sos1 and Abil in pancreatic cancer. Pancreatology 62: A19.

27. Chang YL, Ho BC, Sher S, Yu SL, Yang PC (2015) miR-146a and miR-370 coordinate enterovirus 71-induced cell apoptosis through targeting SOS1 and GADD45 $\beta$ ). Cell Microbiol 17: 802-818.
28. Tartaglia M, Pennacchio A, Zhao C, Yadav KK, Fodale V (2007) Gain-offunction SOS1 mutations cause a distinctive form of Noonan syndrome. Nature Genetics 39: 75-79.

29. Pierre S, Bats AS, Coumoul X (2011) Understanding SOS (Son of Sevenless). Biochem Pharmacol 82: 1049-156.

30. Andreyev HJ, Norman AR, Cunningham D, Oates J, Dix BR, et al. (2001) Kirsten ras mutations in patients with colorectal cancer: the 'RASCAL II' study. Br J Cancer 85: 692-696.

31. Krens LL, Baas JM, Gelderblom H, Guchelaar HJ (2010) Therapeutic modulation of k-ras signaling in colorectal cancer. Drug Discov Today 15: 502-516.

32. Liu X, Jakubowski M, Hunt JL (2011) KRAS gene mutation in colorectal cancer is correlated with increased proliferation and spontaneous apoptosis. Am J Clin Pathol 135: 245-252.

33. Abubaker J, Bavi P, Al-Haqawi W, Sultana M, Al-Harbi S, et al. (2009) Prognostic significance of alterations in KRAS isoforms KRAS-4A/4B and KRAS mutations in colorectal carcinoma. J Pathol 219: 435-445.

34. Wan PT, Garnett MJ, Roe SM, Lee S, Niculescu-Duvaz D, et al. (2004) Mechanism of activation of the RAF-ERK signaling pathway by oncogenic mutations of B-RAF. Cell 116: 855-867.

35. Davies H, Bignell GR, Cox C, Stephens P, Edkins S, et al. (2002) Mutations of the BRAF gene in human cancer. Nature 417: 949-954.

36. Jeng HH, Taylor LJ, Bar-Sagi D (2012) Sos-mediated cross-activation of wild-type Ras by oncogenic Ras is essential for tumorigenesis. Nat Commun 3: 1168.

37. Ebi H, Costa C, Faber AC, Nishtala M, Kotani H, et al. (2013) PI3K regulates MEK/ERK signaling in breast cancer via the Rac-GEF, P-Rex1. Proc Natl Acad Sci U S A 110: 21124-21129.

38. Manser E, Leung T, Salihuddin H, Zhao ZS, Lim L (1994) A brain serine/ threonine protein kinase activated by Cdc42 and Rac1. Nature 367: 40-46.

39. Frost JA, Steen H, Shapiro P, Lewis T, Ahn N, et al. (1997) Cross-cascade activation of ERKs and ternary complex factors by Rho family proteins. EMBO J 16: 6426-6438.

40. Zehorai E, Yao Z, Plotnikov A, Seger R (2010) The subcellular localization of MEK and ERK--a novel nuclear translocation signal (NTS) paves a way to the nucleus. Mol Cell Endocrinol 314: 213-220.

41. Vossler MR, Hong Yao, Randall D York, Ming-Gui Pan, Caroline S Rim, et al. cAMP Activates MAP Kinase and Elk-1 through a B-Raf- and Rap1Dependent Pathway. Cell 89: 73-82.

42. Ueda Y, Hirai Si, Osada Si, Suzuki A, Mizuno K, et al. (1996) Protein kinase $\mathrm{C}$ activates the MEK-ERK pathway in a manner independent of Ras and dependent on Raf. J Biol Chem 271: 23512-23519.

43. Peyssonnaux C, Eychène A (2001) The Raf/MEK/ERK pathway: new concepts of activation. Biol Cell 93: 53-62.

44. Pierre S, Bats AS, Chevallier A, Bui LC, Ambolet-Camoit A, et al. (2011) Induction of the Ras activator Son of Sevenless 1 by environmental pollutants mediates their effects on cellular proliferation. Biochem Pharmacol 81: 304-313.

45. Shin SY, Rath O, Choo SM, Fee F, McFerran B, et al. (2009) Positive- and negative-feedback regulations coordinate the dynamic behavior of the Ras-Raf-MEK-ERK signal transduction pathway. J Cell Sci 122: 425-435.

46. Douville E, Downward J (1997) EGF induced SOS phosphorylation in PC12 cells involves P90 RSK-2. Oncogene 15: 373-383.

47. Nielsen KH, Papageorge AG, Vass WC, Willumsen BM, Lowy DR (1997) The Ras-specific exchange factors mouse Sos1 (mSos1) and mSos2 are regulated differently: mSos2 contains ubiquitination signals absent in mSos1. Mol Cell Biol 17: 7132-7138. 